\title{
Why do Anesthesiologists Drug Administration Errors?
}

\author{
Thomas R Erdmann ${ }^{1 *}$ and Andrea C Erdmann ${ }^{2}$ \\ ${ }^{1}$ Department of Surgery, Federal University of Santa Catarina, Florianópolis, Brazil \\ ${ }^{2}$ Bachelor of Dental Science, Federal University of Santa Catarina, Florianópolis, Brazil
}

\begin{abstract}
Received: August 02, 2016; Accepted: September 06, 2016, Published: September 09, 2016
*Corresponding author: Thomas Rolf Erdmann, MD, Anesthesiologist Professor, Department of Surgery, Federal University of Santa Catarina, Professora Maria Flora Pausewang street, PO Box 88036-800, Florianópolis, Brazil, Tel: +554-832-0681-83; Fax: +55 48 3721-8354; E-mail: thomaserdmann@hotmail.com
\end{abstract}

\begin{abstract}
Medication error represents a particular challenge for the patient safety in tertiary care. Anesthesiologists should attempt against errors because they represents the unique medical specialty that administrate the drug without another professional conference, easily acting as vectors of adverse events during anesthesia [1]. Some factors expose more than others the susceptibility to undesirable events. This review has the purpose to define the main characteristics of drug administration errors in anesthesia practice. Mesh terms "medication errors", "anesthesia" and "anesthesiologists" were combined during the search for studies used in this review. Anesthesiologists errs during the whole perioperative period, but most cases tend to happen in the morning, at the beginning of anesthesia, with no harm, by in-training providers in all types of surgeries and patients. Errors as incorrect dose and substitution due to distraction and fatigue are more common. Some routines should be incorporated to the experience of the anesthesiologist to facilitate the correct practice.
\end{abstract}

\section{Purpose}

Medication error represents a particular challenge for the patient safety in tertiary care. The overall rate is observed in order of 4.87 errors per 100.000 administrations by pharmacists [2]. Anesthesiologists should attempt against errors because they represents the unique medical specialty that administrate the drug without another professional conference, easily acting as vectors of adverse events during anesthesia [1].

Drug administration demands whole attention in the perioperative period, but not always respected in anesthesia practice. In a recent study, adverse events occurs in 1 of 3 anesthetics, medication error in 1 of 2 anesthetics and one of them in 1 of 20 perioperative medication administration [3]. One estimation shows that each anesthesiologist errs seven times a year and causes damage to patients twice over a career [4], representing high and unacceptably common situations.

Medication error is defined as a failure to execute an action in the drug administration process or the use of an incorrect plan or action to achieve a patient care aim [5] and an adverse drug event as patient harm or injury due to administration of a drug [6]. The steps of the administration process include requesting medication from pharmacy, dispensing the medication from the pharmacist to the anesthesiologist, preparing the medication (e.g., aspiration, dilution), administering the drug to reach the patient, documenting in the anesthesia information management system and monitoring the vital signs or patient exams after the administration [3]. According to the working system, errors can be divided in active failure, caused by people in direct contact to the patient, and latent condition, due to the system. Also, errors can be classified as due to omission (failure to perform an appropriate action) and commission (perform an inappropriate action) [7].

At most cases, medication errors occurs at administration stage (53\%), followed by prescription (17\%), preparation (14\%) and transcription (11\%) [8]. Some factors expose more than others the susceptibility to undesirable events. Leading the causes in anesthesia, medication errors far exceeds disconnection of the breathing circuit, the second cause of adverse events [9]. This review has the purpose to define the main characteristics of drug administration errors in anesthesia practice.

Mesh terms "medication errors", "anesthesia" and "anesthesiologists" were combined during the search for studies used in this review. Related studies to them were also used.

\section{Type of Errors}

Errors like syringe swaps, drug ampoule swaps, overdose or incorrect drug choices were cited $[10,11]$, but were also found more detailed as incorrect route (administration of a drug by another route), incorrect dose (unwanted concentration, amount or infusion rate), insertion (drug administered in unwanted time), replacement (administration of a drug different from the indented one), repetition (readministration of a drug due to prior administration uncertainty) and omission (a forgotten/nonadministered drug) [1].

Different results were observed in the studies. In Orser, et al. [10] self-reports study; the commonest cited errors were syringe 
swaps (70.4\%) and misidentification of the label (46.8\%). Yamamoto, et al. [11] retrospectively analyzed an amount of (27454) anesthesia cases during previous 8 years, with only 61 administration errors, mainly overdose (25\%), followed by substitution (23\%) and omission (21\%), also observed in another large prospective observational study, as incorrect dose (36.5\%), substitution (25\%) and omission (19.2\%) [9]. Recent prospective study realized during 277 operations observed higher prevalence of labeling error (24.2\%), followed by dose error (22.9\%) and omission (17.6\%) [3] that corroborates another recent study about one Brazilian State anesthesiologist's errors [1].

\section{Contributing factors to errors}

Distraction/fatigue was the commonest leading factor to errors, followed by misread label, pressure to proceed and improper storage, respectively. Other evaluated items were lack of knowledge or experience, unfamiliar vial or ampoule, inadequate communication among anesthesiologists, equipment malfunction, equipment not available and others $[1,12]$. Cooper, et al. [13] cited factors with similar terms, but at most cases related to inadequate total experience.

Despite Orser, et al. [10] describes $94.9 \%$ of respondents anesthesiologists reports most of the time or always read the label, when asked about the single most important feature of the label, $50.7 \%$ identify colors, $26.9 \%$ identify more than 1 feature on the label, $7.5 \%$ drug name, $7.1 \%$ shape of container, $3.4 \%$ size of container and below $1 \%$ syringe codes, acting as contributing factors.

\section{Time of errors}

For the period of day that error cases occurred, Erdmann, et al. [1] described higher incidence in the morning (32.7\%), in the afternoon (21.8\%) and lower in the evening (16.3\%). Fasting, et al. [14] observed that 56 of 63 drug errors occurred in day shifts. No other related data were found in the reviewed studies.

\section{Perioperative period of errors}

A unique study described the most frequent anesthesia period of error. Errors were most reported by anesthesia providers during maintenance of anesthesia (49\%; 42\%), followed by induction of anesthesia (30.9\%; 28\%) [1,14], extubation $(12.7 \%)$, pre anesthetic $(5.5 \%)$ and post anesthetic periods (1.8\%) [1]. Nanji et al. (2016) found that $53.9 \%$ of events (including medication error and adverse drug event) occurred within 20 minutes of induction period [3].

\section{Drug Involved}

Observational studies observed higher incidence of medication errors in administration of sedatives (propofol) and opioids (fentanyl) were related to $47.4 \%$ of incorrect dose errors $[3,12]$; steroids (dexamethasone) and vasoactive medications were related to $46.2 \%$ of substitution errors [12]. On the other hand, Yamamoto, et al. [11] observed antibiotics as the most frequently involved agents (23\%), followed by muscle relaxants $(15 \%)$ and vasopressors $(15 \%)$ in overall drug administration errors during anesthesia.

\section{Surgery type}

If considered the surgery type, different results were observed. Vascular surgeries are on top 2 most common risk of medication errors (6.8\%; $1.32 \%)$, following general surgery (7.7\%) [3] or pediatrics (1.69\%) [12] in different studies. Thoracic $(5.9 \% ; 1.00 \%)$, orthopedic $(5.5 \% ; 0.56 \%)$ and other abdominal surgeries are also relevant, but without statistically significant association $[3,12]$.

\section{Anesthesia type}

Nanji, et al. [3] observed 5.5\% events (including adverse events) in general anesthesia versus $4.6 \%$ in sedations, without significant difference between event rates $(p=0.52)$. Under neuraxial anesthesia, 7\% of respondents reported drug administration error by this route [1].

ASA classification: Cooper et al. (2012) analyzed the relative risk reported in cases of medication error/pre-error of 8777 anesthesia involving ASA III (0.81\%) compared with ASA I $(0.28 \%)$ and ASA II $(0.43 \%)$ patients, with statistically significant difference under ASA III and ASA I patients ( $p=0.001)$ [12].

In a recent study, Nanji, et al. [3] described $2.9 \%$ of medication errors in ASA I physical status score patients, $4.1 \%$ in ASA II, $4.7 \%$ in ASA III and $7.1 \%$ in ASA IV patients, with no statistically significant association $(\mathrm{p}=0.56)$.

Body mass index, duration of procedure and medication administrations: No significant association existed when compared Body Mass Index (BMI), defined as normal, overweight and obese, with medication errors [3].

Procedures duration analyzed as less than $1 \mathrm{~h}(3.0 \%$ of medication errors), $1-3 \mathrm{~h}(4.2 \%), 3-6 \mathrm{~h}(4.1 \%)$ and more than $6 \mathrm{~h}$ (7.3\%) reveal that longer procedures, especially greater than 6 hours, had higher medication error rates $(p<0.0001)$ than shorter procedures [3].

Despite 13 or more medication administrations had significant difference of adverse drug events rates between 12 or fewer $(1.8 \%)$ and 13 or more $(2.8 \%)(p=0.002)$, no significant association was observed between 12 or fewer (4.8\%) and 13 or more $(3.9 \%)(p=0.11)[3]$.

No other related data to BMI, duration of procedure and number of medication administrations were found in the reviewed studies.

\section{Experience of provider}

In a study with anesthesia providers (in-training and experienced, nurses and physicians), statistically significant difference was observed between comparison of provider-intraining group $(0.74 \%$ or $1: 135$ anesthetics) and experienced provider group $(0.37 \%$ or $1: 272$ anesthetics) with a relative risk of 2 ( $p=0.0087$ ) for the distribution of reported medication errors/pre-errors [12]. Considering overall events (medication errors and adverse events), no significant difference existed between house staffs (5.1\% event rate), nurse anesthetists $(5.5 \%)$ and attending anesthesiologists $(4.5 \%)(p=0.79)$ [3]. 


\section{Injury severity}

Negative outcomes were stratified in different types in the reviewed studies. Most cases of medication error caused no harm or minor consequence. Major consequence with reversible or irreversible damage (including cardiac arrest, stroke and permanent injury) was reported by no more than $8.75 \%$ of medication errors $[1,3,10-12,14]$.

No fatal errors were described in most studies $[1,3,11,12]$. Only one study reported a number higher than $0.4 \%$ of deaths, but included all type of anesthesia/equipment errors [9]. Orser, et al. [10] reported 4 fatal medication errors $(<0.4 \%)$ resulted from overdose of ketamine due to misidentification of a vial, administration of norepinephrine instead of fentanyl, potassium chloride solution used to dilute an antibiotic and peritoneal dialysate infused in another route.

\section{Medication Shortage and Safety in Anesthesia}

Shortage of perioperative drugs are inversely related to patient care. The anesthesiologist should caution on lack or return of medications, as a newer concentration or changing the anesthesia technique, in adaptation to avoid administration errors [15].

As reported by Ogboli-Nwasor, [16] similar ampoules induce drug administration errors, when in different medications and different doses. To avoid these cases and for safety in anesthesia, system contributing factors are already used, as color- and barcode [16-19], and a double-check by one person or "four-eyecheck" by two qualified practitioners [20], when possible. Other safety system is syringe, needle or other devices connectors that connect with a specific kind of route injectors [20].

Jensen et al. [21] summarized potential strategies to prevent drug administration errors during anesthesia as:

- Read the label of medication ampoule or syringe before the medication drawn up or injection

- Syringes need to be labeled

- Organize the position of ampoules and syringes, and caution with dangerous medications on anesthesia workstation

- Double-check medications before administration

- Report any drug administration error during anesthesia

- Appoint a dedicated pharmacist for the operating room

- Avoid the use of similar ampoules and syringes of medications

- Use prefilled syringes for medications, if possible

- Only the anesthesia provider who will administer the medication should drawn up and label the medication

- Standard color code by class of medication should be agreed
Tobias, et al. [22] mentioned the "5 rights of medication administration" to follow, as:

- The right patient

- The right drug

- The right dose

- The right time

- The right route

Nowadays, despite the different workplaces, these factors are seen on anesthesia practice in most countries and must be worldwide introduced.

\section{Conclusions}

Anesthesiologists errs during the whole perioperative period, but most cases tend to happen in the morning, at the beginning of anesthesia, with no harm, by in-training providers in all types of surgeries and patients. Errors as incorrect dose and substitution due to distraction and fatigue are more common. Some routines should be incorporated to the experience of the anesthesiologist to facilitate the correct practice.

\section{Declarations}

The authors declare that there is no conflict of interest regarding the publication of this paper.

\section{Disclaimer}

The view expressed in this paper represents the views of the authors and are not officially endorsed by the institution.

\section{Source of support}

This study was funded by the authors.

\section{References}

1. Erdmann TR, Garcia JH, Loureiro ML, Monteiro MP, Brunharo GM. Profile of drug administration errors in anesthesia among anesthesiologists from Santa Catarina. Rev Bras Anestesiol. 2016;66(1):105-110. doi:10.1016/j.bjan.2014.06.004

2. Gorbach C, Blanton L, Lukawski BA, Varkey AC, Pitman EP, Garey KW. Frequency of and risk factors for medication errors by pharmacists during order verification in a tertiary care medical center. Am J Health Syst Pharm. 2015;72(17):1471-1474. doi:10.2146/ajhp140673

3. Nanji KC, Patel A, Shaikh S, Seger DL, Bates DW. Evaluation of Perioperative Medication Errors and Adverse Drug Events. Anesthesiology. 2016;124(1):25-34. doi:10.1097/ ALN.0000000000000904

4. Glavin RJ. Drug errors: consequences, mechanisms, and avoidance. $\mathrm{Br}$ J Anaesth. 2010;105(1):76-82. doi:10.1093/bja/aeq131

5. Rothschild JM, Landrigan CP, Cronin JW, Kaushal R, Lockley SW, Burdick E, et al. The Critical Care Safety Study: The incidence and nature of adverse events and serious medical errors in intensive care. Crit Care Med. 2005;33(8):1694-1700.

6. Bates DW, Cullen DJ, Laird N, Petersen LA, Small SD, Servi D, et al. Incidence of adverse drug events and potential adverse drug events. Implications for prevention. ADE Prevention Study Group. JAMA. 1995;274(1):29-34 
7. Kothari D, Gupta S, Sharma C, Kothari S. Medication error in anaesthesia and critical care: A cause for concern. Indian J Anaesth. 2010;54(3):187-192. doi:10.4103/0019-5049.65351

8. Lampert T, Thamm M. Consumption of tobacco, alcohol and drugs among adolescents in Germany. Results of the German Health Interview and Examination Survey for Children and Adolescents (KiGGS). Bundesgesundheitsblatt Gesundheitsforschung Gesundheitsschutz. 2007;50(5-6):600-608. doi:10.1007/s00103-007-0221-y

9. Cooper JB, Newbower RS, Kitz RJ. An analysis of major errors and equipment failures in anesthesia management: considerations for prevention and detection. Anesthesiology. 1984;60(1):34-42.

10. Orser BA, Chen RJ, Yee DA. Medication errors in anesthetic practice: a survey of 687 practitioners. Can J Anaesth. 2001;48(2):139-146. doi:10.1007/BF03019726

11. Yamamoto $\mathrm{M}$, Ishikawa $\mathrm{S}$, Makita $\mathrm{K}$. Medication errors in anesthesia: an 8-year retrospective analysis at an urban university hospital. J Anesth. 2008;22(3):248-252. doi:10.1007/s00540-008-0624-4

12. Cooper L, DiGiovanni N, Schultz L, Taylor AM, Nossaman B. Influences observed on incidence and reporting of medication errors in anesthesia. Can J Anaesth. 2012;59(6):562-570. doi:10.1007/s12630012-9696-6

13. Cooper L DN, Schultz L, Taylor RN, Nossaman B. Human factors contributing to medication errors in anaesthesia practice. ASA 2009:A614.

14. Fasting S, Gisvold SE. Adverse drug errors in anesthesia, and the impact of coloured syringe labels. Can J Anaesth. 2000;47(11):10601067. doi:10.1007/BF03027956
15. De Oliveira GS, Jr., Theilken LS, McCarthy RJ. Shortage of perioperative drugs: implications for anesthesia practice and patient safety. Anesth Analg. 2011;113(6):1429-1435. doi:10.1213/ANE.0b013e31821f23ef

16. Ogboli-Nwasor E. Medication errors in anaesthetic practice: a report of two cases and review of the literature. Afr Health Sci. 2013;13(3):845849. doi:10.4314/ahs.v13i3.46

17. Jelacic S, Bowdle A, Nair BG, Kusulos D, Bower L, Togashi K. A System for Anesthesia Drug Administration Using Barcode Technology: The Codonics Safe Label System and Smart Anesthesia Manager. Anesth Analg. 2015;121(2):410-421. doi:10.1213/ANE.0000000000000256

18. Nolen AL, Rodes WD 2nd. Bar-code medication administration system for anesthetics: effects on documentation and billing. Am J Health Syst Pharm. 2008;65(7):655-659. doi:10.2146/ajhp070167

19. Merry AF, Webster CS, Mathew DJ. A new, safety-oriented, integrated drug administration and automated anesthesia record system. Anesth Analg. 2001;93(2):385-390.

20.Staender S. Better look twice--medication errors in anaesthesia and how to avoid them. Rev Esp Anestesiol Reanim. 2011;58(6):335-336.

21. Jensen LS, Merry AF, Webster CS, Weller J, Larsson L. Evidencebased strategies for preventing drug administration errors during anaesthesia. Anaesthesia. 2004;59(5):493-504. doi:10.1111/j.13652044.2004.03670.x

22. Tobias JD YG, Gupta SK, Jain G. Medication errors: A matter of serious concern. Anaesth Pain\& Intensive Care. 2013;17(2):111-114. 